\title{
The Autonomous Pathogen Detection System
}

J. M. Dzenitis, A. J. Makarewicz

January 26, 2009

Chapter in Book: The Microflow Cytometer 
This document was prepared as an account of work sponsored by an agency of the United States government. Neither the United States government nor Lawrence Livermore National Security, LLC, nor any of their employees makes any warranty, expressed or implied, or assumes any legal liability or responsibility for the accuracy, completeness, or usefulness of any information, apparatus, product, or process disclosed, or represents that its use would not infringe privately owned rights. Reference herein to any specific commercial product, process, or service by trade name, trademark, manufacturer, or otherwise does not necessarily constitute or imply its endorsement, recommendation, or favoring by the United States government or Lawrence Livermore National Security, LLC. The views and opinions of authors expressed herein do not necessarily state or reflect those of the United States government or Lawrence Livermore National Security, LLC, and shall not be used for advertising or product endorsement purposes. 
Chapter title: The Autonomous Pathogen Detection System

Chapter authors: J. M. Dzenitis and A. J. Makarewicz

Book title: The Microflow Cytometer

Book editors: J. Kim and F. Ligler

LLNL-JRNL-410104, Book chapter

$3 / 16 / 2009$

This work was performed under the auspices of the U.S. Department of Energy by Lawrence Livermore National Laboratory in part under Contract W-7405-Eng-48 and in part under Contract DE-AC5207NA27344.

This document was prepared as an account of work sponsored by an agency of the United States government. Neither the United States government nor Lawrence Livermore National Security, LLC, nor any of their employees makes any warranty, expressed or implied, or assumes any legal liability or responsibility for the accuracy, completeness, or usefulness of any information, apparatus, product, or process disclosed, or represents that its use would not infringe privately owned rights. Reference herein to any specific commercial product, process, or service by trade name, trademark, manufacturer, or otherwise does not necessarily constitute or imply its endorsement, recommendation, or favoring by the United States government or Lawrence Livermore National Security, LLC. The views and opinions of authors expressed herein do not necessarily state or reflect those of the United States government or Lawrence Livermore National Security, LLC, and shall not be used for advertising or product endorsement purposes. 


\title{
CHAPTER 17
}

\section{THE AUTONOMOUS PATHOGEN DETECTION SYSTEM}

\author{
John M. Dzenitis and Anthony J. Makarewicz \\ Lawrence Livermore National Laboratory \\ 7000 East Avenue, Livermore California, 94550 \\ E-mail: john.m.dzenitis@llnl.gov
}

\begin{abstract}
We developed, tested, and now operate a civilian biological defense capability that continuously monitors the air for biological threat agents. The Autonomous Pathogen Detection System (APDS) collects, prepares, reads, analyzes, and reports results of multiplexed immunoassays and multiplexed PCR assays using Luminex@ xMAP technology and flow cytometer. The mission we conduct is particularly demanding: continuous monitoring, multiple threat agents, high sensitivity, challenging environments, and ultimately extremely low false positive rates. Here, we introduce the mission requirements and metrics, show the system engineering and analysis framework, and describe the progress to date including early development and current status.
\end{abstract}

\section{Importance}

Biological terrorism is an increasing concern: biological technology is advancing in capability while also becoming more accessible, and terrorist activities are increasing in number, scale, and diversity. Dissemination of biological threat agents as aerosols is a particular menace because of the ease of widespread dispersal and the effectiveness of infection by inhalation. Lawrence Livermore National Laboratory (LLNL) developed the Autonomous Pathogen Detection System (APDS) for the U.S. Department of Energy (DOE) and Department of Homeland Security (DHS) as a means of detecting biological threat agents in the 
air. The APDS was developed for high-risk locations to reach the bestachievable combination of competing characteristics such as speed, selectivity, sensitivity, numbers of agents, and cost. For example, while fast detection can prevent exposures and allow the most effective medical treatment of people already exposed, ${ }^{1}$ false positive results must also be extremely low in a civilian setting. The APDS is the first actionable autonomous detector ${ }^{2}$ component of the DHS's BioWatch Program. ${ }^{3}$

The APDS instrument will be of interest to readers of this book because the analytical core of the instrument is a flow cytometer. In the initial stages of development, compact and advanced flow cytometers provided fast and sensitive detection of biological agents. In subsequent development, the more conventional cytometry approach was replaced with a Luminex assay platform and flow cytometer to allow detection of many agent signatures at once. The compact flow cytometer is one subsystem in the larger system which includes aerosol collection, biological reagents, sample preparation, result analysis, field packaging, communications, maintenance support, and remote monitoring. This chapter does not focus on the flow cytometer itself, but instead provides an overview of the characteristics, progress, and critical issues of an advanced, fielded system utilizing an automated flow cytometry system.

\section{Characteristics of pathogen detection systems}

\subsection{Mission and metrics}

The fundamental mission of pathogen detection systems is to provide information that saves lives. For DHS's BioWatch Program, the system needs to continuously sample the air at a location over many months and produce regular reports that either the air is uncontaminated or that there is potentially a biological threat agent present. This is true of both the manual system, where dry filters are transported daily to Laboratory Response Network laboratories for analysis and reporting, ${ }^{3}$ and the APDS, where the analysis is performed in the field and reported via a network. The response to positive signals could include facility 
operators closing a facility to limit further exposure, response crews in personal protective equipment searching for sources and performing manual sampling, public health laboratories performing characterization testing, medical testing and countermeasures, and other actions. Other operational systems with similar missions include the U.S. Postal Service's Biohazard Detection System ${ }^{4}$ and the Department of Defense's (DoD's) Joint Biological Point Detection System (JBPDS). ${ }^{5}$ However, the APDS mission differs in requirements including monitoring for the public, continuous operations, number of agents, and the stakeholders involved.

The overall metrics for the detection system depend a great deal on the stakeholders. Here, the term "stakeholders" is used instead of "users" to focus on those who act on the system's biological information. From this perspective, the personnel who actually keep the equipment running are viewed as part of the system. The DHS commissioned an expert panel in 2006 to examine metrics for biological detection systems from the stakeholder perspectives, not just technical perspectives. The panel included local authorities (facility operators, public health officials, and law enforcement personnel) and national authorities (DHS, Centers for Disease Control and Prevention [CDC], DoD, and the Postal Service). The details of the study are not public information, but some qualitative aspects can be described here.

Ultimately, the utility of the system to the stakeholders should be described in terms of cost versus benefit, where cost includes direct, indirect, and opportunity costs, and benefit includes saving lives. More accurately, and perhaps more coldly, the system should be analyzed in terms of Net Present Value, including the probability of an attack and the financial value of a human life. As a step towards the larger picture, cost and benefit categories were used to organize the quantifiable characteristics of the system as follows:

\section{Benefit metrics}

- Detect agents of interest: agent types, agent panel, number of signatures, time to add an assay, probability of detection, and operational availability. 
- Detect in the required environments: environments of interest, temperature range, humidity range, particle tolerance, battery capability, and additional factors such as electromagnetic interference.

- Detect at effective levels: sensitivity as limit of detection.

- Enable effective response: probability of false alert, probability of actionable false positive, selectivity, data accessibility, remote system diagnostics, data security, physical security, biohazard security, data time-stamping capability, data archiving duration, and sample archiving capability.

- Report on an effective timescale: sampling period and time to results.

Cost metrics

- Direct acquisition cost.

- Direct operation cost.

- Indirect operation cost to the local stakeholders: local interoperability, facility labor requirement, biological monitor labor requirement, maintenance interval, mean time between failure, power, size, visual impact, and noise.

Some of the metrics affect multiple areas (e.g., probability of false positive affects effective response and indirect operation cost), but were placed in what was judged to be their primary category. In a full costbenefit analysis, the effects would just apply quantitatively as appropriate. Subsequent work with the stakeholders for the particular APDS application established specific threshold values, and more stringent goal values, for these metrics. Although this limited effort fell short of a cost-benefit or net-present-value calculation, having quantified values with stakeholder agreement was an important step. We will point out some of the less obvious results.

Of all of the parameters, probability of false actionable results was the most stringent: the critical locations that would be monitored would also have a tremendous impact if shut down, and clearing a location to reopen after a biological alert would take hours because of the sampling and testing required. There is a relationship between sensitivity, 
probability of detection, and probability of a false positive, often discussed as the Receiver Operating Characteristic (ROC). ${ }^{6}$ In the present mission, the stakeholders felt that the false-positive rate needs be set to a very low level that they can tolerate in operations, and the resulting sensitivity is either acceptable or not. They do not view it as a trade-off.

The ability to detect numerous different agent signatures in every sample was important for several reasons. First, several different agents could be used in the attack, so of all the likely possibilities need to be represented to detect the agents of interest. Second, the false-positive rate must be extremely low, and having multiple independent signatures per agent helps greatly. However, having many signatures can quickly drive up the acquisition and operation costs if many separate analyses need to be done.

Some of the traditional parameters had little meaning by themselves, but primarily affected other metrics. For example, Mean Time Between Failures (MTBF) is a key characteristic, but in itself is not a cost. Its effects are in operational availability, in direct cost for labor and materials, and potentially in indirect cost of inconvenience to the facility operations.

Finally, there is often a focus on direct acquisition cost of complex instrumentation. However, for this type of system and mission, the yearly operation costs will be on the same scale as the acquisition cost, so they need to be considered together.

\subsection{System engineering and analysis}

The steps in a detection process can be organized in a number of ways. We will use the following steps for the first level:

- Collect the sample

- Prepare the sample for detection

- Read the prepared sample

- Analyze the results

- Report the result 
An example schematic diagram of the process flow over time for a pathogen detection system is shown in Figure 1. The system repeatedly executes the steps of collect, prepare, read, analyze, and report. In this example, the collection is continuous but split into segments. After the step Collect 1 ends, Prepare 1 begins on that collected sample, while the next collection step begins in parallel. The other processing steps are assumed to be sequential here, so in this case a release that occurs during Collect 2 is reported near the end of Collect 3 and leads to a response action.

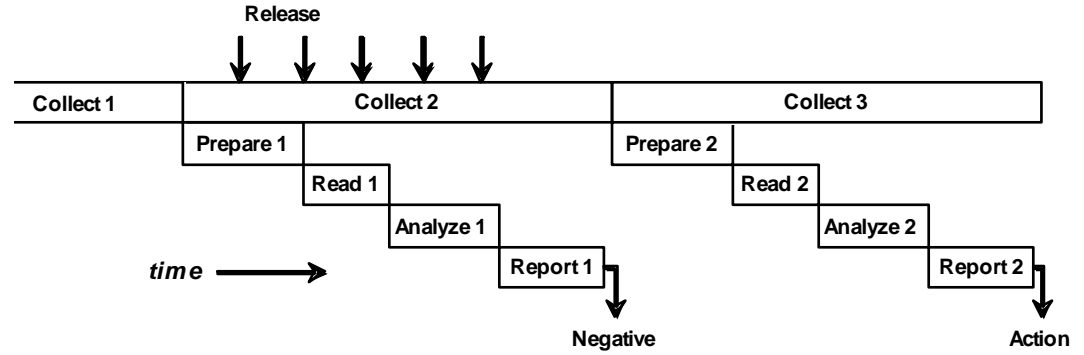

Figure 1. A schematic diagram of the overall process flow over time for a pathogen detection system. In this example mode of operation, the collection is continuous but split into distinct segments, and all of the other processing steps are sequential. After the step Collect 1 ends, Prepare 1 begins on that collected sample, while the next collection step begins in parallel. A release that occurs during Collect 2 is reported during Collect 3 . The relative times are not to scale.

\section{Process analysis approach}

It is useful to have a basic mathematical framework to describe the functions of the various steps in the process train. This will allow us to illustrate the important characteristics of the steps, although we will not go into the quantitative parameters here. The mass balance equation that we use is written for rate of change of a number of detectable targets, $n$, in a control volume as

$$
\frac{d n_{C V}}{d t}=\dot{n}_{i n, C S}-\dot{n}_{\text {out }, C S}+\dot{n}_{\text {form }, C V}
$$

where $\dot{n}$ is a flow or formation rate of number of targets, and $C V$ and $C S$ indicate the conceptual control volume and surface enclosing the process 
step. The targets could be cells, spores, protein epitopes, DNA copies, colony forming units (CFUs) et cetera, depending on the detection technique. The last term in Equation 1 represents formation (or if negative, loss) of detectable targets. This is not creation or destruction of mass but rather transformation to or from a detectable form. The usefulness of the concept will be evident later.

Often the processes are carried out batch-wise instead of continuously so we use an integrated form of Equation 1. Also, in order to avoid losses through aliquoting or sub-sampling, it is as desirable to maintain a high concentration as it is to maintain copies of targets. Therefore, we can evaluate the performance of the processing steps as

$$
\frac{c_{\text {out }}}{c_{\text {in }}}=\frac{n_{\text {out }} / V_{\text {out }}}{n_{\text {in }} / V_{\text {in }}}
$$

so that the volumes in and out of the step are part of the rating of the process, along with the number of targets in and out. In the unlikely case that there is no downstream volume limitation, then it may be appropriate not to consider the volume changes and only consider number of targets or efficiency.

\section{Collect}

The aerosol collector converts the environmental material into an internal sample that can be manipulated. The net rate of collection that comes from Equation 1 is

$$
\frac{d n_{\text {coll }}}{d t}=Q_{\text {coll }} \eta_{\text {coll }} c_{\text {in }}-k_{\text {loss }} n_{\text {coll }}
$$

where $n_{\text {coll }}$ is the number of targets in the collector, $Q_{\text {coll }}$ is the collector flow rate, $\eta_{\text {coll }}$ is the collection efficiency, $c_{i n}$ is the target concentration in the air into the collector, and $k_{\text {loss }}$ is a loss rate constant.

The first term on the right of Equation 3 is the collection rate. Collection efficiency is important in system design, but the maximum efficiency for any collector is unity while the flow rate for some collectors can be much larger than others. For this reason we emphasize that the collector flow rate is potentially more important than its efficiency. The APDS instrument uses a liquid-based collection, and we 
value small collection volumes because we want to minimize the material loss from aliquoting downstream. The integrated version of Equation 3 for output liquid concentration considering only the collection term is

$$
c_{\text {out }}=\left(\frac{Q_{\text {coll }} \eta_{\text {coll }}}{V_{\text {coll }}}\right) \int c_{\text {in }} d t=\dot{X}_{\text {coll }} \int c_{\text {in }} d t
$$

if everything is constant over time except for the air concentration. The $\dot{X}$ term is the concentration rate and has units such as (targets/L liquid)/ (targets/L air)/ min, more casually written $\mathrm{min}^{-1}$. This parameter is the best single measure of collection performance.

The last term on the right of Equation 3 is the loss rate, written here with the assumption of first-order decay. This term accounts for the fact that collected targets may be lost through mechanisms like reaerosolization or degradation, and decreases the output concentration to be less than Equation 4 . The loss rate may be significant for long collection times or when the air concentration is low.

Prepare: Lyse

Lysis is a sample preparation step that is useful in some cases to convert bound, inaccessible target material to detectable target material. Here, Equation 2 becomes

$$
\frac{c_{\text {out }}}{c_{\text {in }}}=\frac{n_{\text {in }}+\eta_{\text {lyse }} n_{\text {bound }}}{n_{\text {in }}} \frac{V_{\text {in }}}{V_{\text {out }}}=\left(1+\eta_{\text {lyse }} X_{\text {bound }}\right) \frac{V_{\text {in }}}{V_{\text {out }}}
$$

where $n_{\text {bound }}$ is number of bound or inaccessible targets in, $X_{\text {bound }}$ is the ratio of bound to free targets in, and $\eta_{\text {lyse }}$ is the lysis efficiency. We write the performance in this way to point out that the utility of the lysis step depends on not just the efficiency but also on the sample type. Since efficiency is at most unity, the lysis cannot have much effect if the bound target ratio is significantly less than unity. We have tested bacterial spore preparations with $X_{\text {bound }}$ between 0.0001 and 100 in our laboratory, but wider ranges are possible.

Prepare: Extract 
Extraction is a sample preparation step with the goals of purifying and, in some cases, concentrating the material. From the perspective of the downstream analysis, purification creates more detectable target in the sample by removing interfering material. Thus, the measure of the utility of the purification should depend on the analysis. We can use a factor improvement in sensitivity $X_{e x t}$ to attribute the benefit from purification to the extraction step, and an extraction efficiency $\eta_{\text {ext }}$ to account for potential loss of material. This gives

$$
\frac{c_{\text {out }}}{c_{\text {in }}}=\eta_{\text {ext }} X_{\text {ext }} \frac{V_{\text {in }}}{V_{\text {out }}} .
$$

We use this form to consider the combined effects of purification and concentration (or dilution), tempered by the efficiency. If the sample is completely undetectable without the purification, $c_{\text {in }}$ by the definition here as detectable material would be zero, and a different interpretation should be used instead. Extraction is then a requirement and not a choice, and it is characterized just by volume ratio and efficiency.

Prepare: Amplify

PCR, reverse-transcriptase PCR, and other nucleic acid amplification methods form more copies of detectable target. The efficiency should be written such that the number of targets would optimally double with each cycle. At the earlier stages of the reaction the efficiency can be constant over a number of thermal cycles, but more generally for PCR with efficiency $\eta_{i}$ for a cycle number $i$ the increase is

$$
\frac{c_{\text {out }}}{c_{\text {in }}}=\frac{V_{\text {in }}}{V_{\text {out }}} \prod_{i}\left(1+\eta_{i}\right) \text {. }
$$

The efficiency rolls off for later steps as primers, nucleotides, competitive binding, or enzyme activity becomes limiting. In the extreme case, the concentration of targets out is not strongly dependent on the number of cycles or the concentration of targets in because the reaction becomes limited by the other factors. Different PCR signatures can have different sets of efficiencies and different detection limits. Those 
differences can be important operationally when multiple signatures target a single agent.

The amplification possible in the PCR is tremendous and detection from a single copy into the PCR can be approached. The enzyme and primers for a PCR can be expensive, and there is a drive to make the reaction and input volumes as small as possible. For small volumes or low concentrations, the implicit assumption of a continuum in Equation 7 hits a statistical limit of a Poisson process. ${ }^{7}$ If the average number of targets per aliquot is unity, there is a $37 \%$ probability that any given aliquot has no targets at all. To drive that probability down to $1 \%$ for a more acceptable probability of detection, the input concentration and volume must average at least five targets into the reaction, or

$$
n_{\text {in }}=c_{\text {in }} V_{\text {in }}>5 \text { targets. }
$$

Equation 8 shows that as the input volume is decreased, the required input concentration must be correspondingly increased. For example, a 6 $\mu \mathrm{L}$ input sample volume requires a concentration greater than 833 targets/mL. This is not necessarily CFUs, as there are generally more nucleic acid sequence copies than CFUs. The volumetric sampling effect on sensitivity is one of the reasons that "millifluidics" (millimeter length scales) of the type described here are more appropriate than true microfluidics: a $10 \mathrm{pL}$ input volume ${ }^{8}$ requires $5 \times 10^{8}$ targets $/ \mathrm{mL}$. Preconcentration steps or analyzing many representative subsamples can decrease the impact of this requirement.

\section{Prepare: Bind}

In the multiplexed antibody and nucleic-acid assays on the Luminex platform, binding of the biological targets to the bead and subsequent labeling with fluorescent targets must occur so the material can be detected. For the antibody assays we use, the steps are (1) bind the antigen to the bead conjugated with the corresponding antibody, (2) bind the biotinylated labeling antibody to the antigen-bead complex, and (3) label the antibody-antigen-bead complex with streptavidinphycoerythrin. ${ }^{9}$ For the DNA assays we use, the steps are (1) bind the biotinylated PCR amplicon to the bead conjugated with the 
corresponding DNA probe and (2) label the amplicon-bead complex with streptavidin-phycoerythrin. ${ }^{10}$ In both cases, there is a fundamental change in medium from aqueous solution to bead surface. For each signature in the multiplexed assay, we can express the average output concentration per bead as

$$
c_{b}^{\prime}=\eta_{\text {bind }} c_{\text {in }} V_{\text {in }} / n_{b},
$$

where $c_{b}^{\prime}$ is the bead concentration of fluorophore (e.g., molecules of phycoerythrin per bead), $\eta_{\text {bind }}$ is the combined efficiency of all of the binding steps, $c_{\text {in }}$ and $V_{\text {in }}$ are the target concentration and volume in, and $n_{b}$ is the number of labeled Luminex beads that result. The prime notation is used to note the shift from volumetric concentration to bead concentration.

In reality, a distribution of concentrations for the bead population results instead of a single concentration, but the average concentration in Equation 9 serves to illustrate a point: In addition to maximizing the number of targets in and the binding efficiency, there is also an impetus to keep the number of beads low in order to maximize the fluorescence per bead and enhance sensitivity. However, the number of beads needs to be kept high enough for good statistics, as will be discussed below. On the other end of the spectrum, when the input amount is very high, Equation 9 is less useful because the bead concentration asymptotes towards saturation.

\section{Read}

The labeled beads for each signature are read by the Luminex 100 flow cytometer which converts each signature's fluorophore concentration $c_{b}^{\prime}$ into a signal $s$ in Median Fluorescence Intensity (MFI). The dependence of MFI on number of beads in the sample has been analyzed in some detail, ${ }^{11}$ but a general rule-of-thumb is to have at least 50 to 100 beads of each type in an analysis in order to have good reproducibility. If there are approximately 200 beads in a sample, and if the limit of detection is approximately 500 fluorophores per bead, then with perfect binding Equation 9 requires that at least $1 \times 10^{4}$ biological targets would have to enter the binding step. This is readily achieved for PCR due to 
the high target amplification (see Equations 7 and 8) from a concentration of about $1 \times 10^{3}$ targets $/ \mathrm{mL}$. This is more of a restriction for immunoassays, where a $100 \mu \mathrm{L}$ sample would need to provide $1 \times 10^{5}$ targets $/ \mathrm{mL}$. However, the targets do not have to be CFUs: the requirement is mitigated by the fact that there can be multiple biotin targets per antibody, and multiple antibody epitopes per CFU.

\section{Analyze}

After the multiplexed signals have been acquired, the first step of the numerical analysis is determining whether the results meet the criteria for validity. In addition to a requirement for a minimum number of beads of each type, there is also a set of positive and negative controls that must satisfy predetermined thresholds. ${ }^{2,18}$ For multiplexed PCR, the most stringent of these is the positive amplification control (PC), which requires all of the amplification, binding, and reading processes to be executed properly. This prevents false-negative results.

The next step of the analysis is a comparison of the signal results against predetermined thresholds for each signature. For these thresholds to be at a minimum, the values are required to be below a maximum probability of false result, given a signature's baseline and noise behavior, but may also be set higher if a higher LOD is desired for operational reasons.

Finally, there is an analysis of the signature positives against a decision algorithm for alerts. For the multiplexed assays of interest here, a high-level alert requires that multiple signature positives for an agent occur on the same sample. To the extent that the false-signature results are independent, the probability of a false alert will be much less than a single false-signature reaction. If the noise is independent, the probability of a false result from a signature combination is the product of the individual probabilities, for example

$$
p_{f, 123}=p_{f, 1} p_{f, 2} p_{f, 3} .
$$

This form would be modified if there were three of four signatures required or if there is correlation between the signatures' noise. 
Equation 10 shows how the very low false-positive rates required for the mission can be achieved. Three signatures with individual probability of $2 \times 10^{-3}$ would have a combined probability of $8 \times 10^{-9}$. This is the order of stringency that needs to be approached for this application.

\section{Report}

An autonomous detection system must communicate its results quickly so appropriate response actions can be taken. For the BioWatch Program, the local public health officials are the critical link converting technical data into human decisions leading to response, independent of whether the data is from manual or autonomous systems. The autonomous detection instruments must be networked, biological alerts must be reported immediately to mobile messaging devices for response, and biological and maintenance raw data must always be available for remote review.

\section{Associated functions}

In addition to the core process steps of collect, prepare, read, analyze, and report, the system engineering must include functions to sustain these processes. One significant part of this is packaging and hardening against the environment, which includes controlling internal temperature in wide ambient temperature variations, handling dust and precipitation, and operating through temporary power loss and voltage spikes.

Internally, the system needs to perform automatic cleaning, adjustments, and self-monitoring of performance. Externally, there need to be manned support functions of preventative maintenance, corrective maintenance, and monitoring of performance. These, in turn, are part of a larger set of integrated logistics and supply functions for the system on the larger scale. 


\section{Timing}

For the biological process steps described above and shown in Figure 1, the time required for the prepare step dominates read, analyze, and report, which together require only a minute or two. The preparation of a multiplexed immunoassay can take from about 15 to 60 minutes, and the preparation of a multiplexed PCR assay takes from 90 to 150 minutes, depending on the extent of the sample preparation steps. Faster processing of these assays leads to lower sensitivity.

Some tests that do not require such intricate molecular biology can be executed on significantly shorter time frames. However, these other tests have not been able to meet the high sensitivity and extremely low falsepositive rate required for the mission.

\section{Review of progress}

\subsection{Early development}

The reality of the threat of modern chemical and biological terrorism became increasingly apparent at the beginning of the 1990s. The most publicized event was the Aum Shinryko sarin attack in the Tokyo subway in 1995, preceded by an attempted botulinum toxin attack in 1990 and an attempted anthrax attack in 1993. ${ }^{12}$ The APDS project was initiated in 1996 by LLNL as an internally funded project, but in 1997 transitioned to be part of the DOE's Chemical and Biological Nonproliferation Program as that program advanced. The APDS development efforts included a high-performance LLNL flow cytometer called the miniFlo ${ }^{13,14}$, successfully demonstrated at the DoD's 1996 Joint Field Trials III (see Figure 2). This early success contributed to the establishment of the DOE program and of the APDS project. 


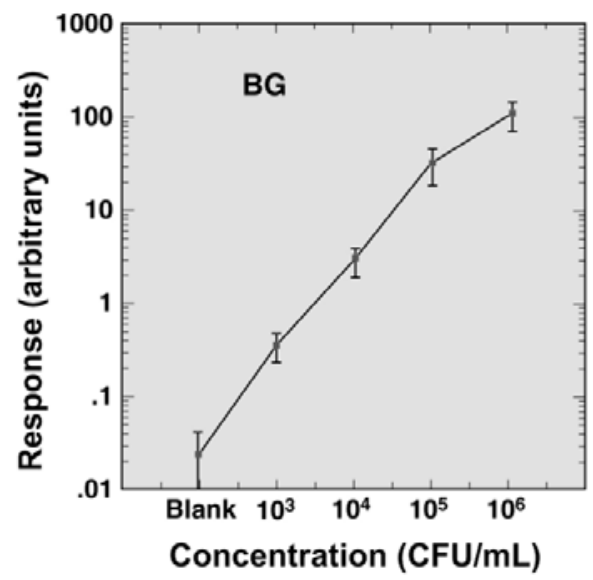

Figure 2. Response of the miniFlo flow cytometer to a biowarfare simulant, Bacillus subtilis var. niger ("BG"), using direct detection of cells by fluorescein-labeled antibodies in the DoD's 1996 Joint Field Trials III at Dugway Proving Ground. The samples were successfully analyzed as unknowns during the trial and later displayed in this format after the samples were unblinded.

The original version of the APDS instrument included the miniFlo flow cytometer and a solenoid valve-based fluidics system (see Figure 3). LLNL began collaborating with Research International Inc. (Monroe, WA) and using an early version of their SASS 2000 wettedwall cyclone aerosol collector. The initial advances in automation of environmental biological detection using immunoassays were achieved in that period. The basic philosophy of using automated fluidics instead of robotics and disposables was established at that time and continues on today. 


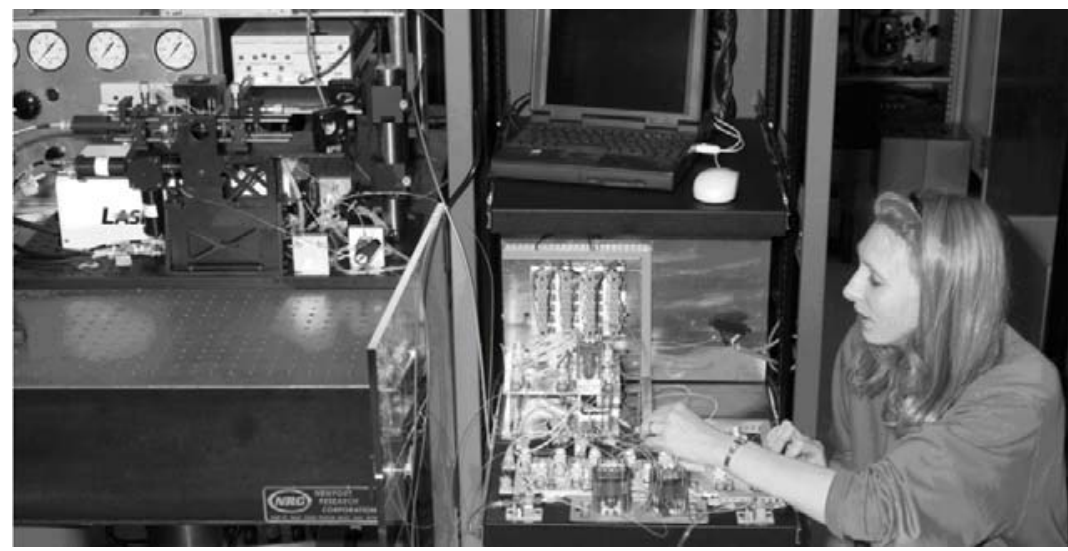

Figure 3. An LLNL engineer works on the fluidics for the original version of the APDS in 1998. The miniFlo microflow cytometer is on the optics table in the left of the photograph.

In 1999, the DOE funded LLNL to undertake revisions to the APDS (then termed APDS II and later APDS100) to extend the capabilities of the system. This incorporated related work at LLNL in this area funded by the DoD. The core change was in the assay preparation and reading format from direct staining of cells and the miniFlo to Luminex Corporation's (Austin, TX) bead sandwich assay platform including the Luminex 100 microflow cytometer reader. The Luminex technology is described in more detail by Roth in the chapter "Luminex System" in this book. There were two key system-level drivers for making this change to the Luminex platform: (1) being able to multiplex assays up to 100 channels per analysis, and (2) having the capability to perform both immunoassays and nucleic-acid assays on the same platform. The multiplexing capability is important because, as noted in the metrics description, many different threat agents could be used and thus many assays should be run at once. Multiplexing gives the ability to perform the analysis without the cost and reliability problems of running many separate analyses. The ability to run both immunoassays and nucleicacid assays is important for orthogonal detection and the most sensitive and specific assay combination for different threat agents. 
The other core subsystems set at that time, and still in use now in upgraded versions, were the aerosol collector and the fluidics module. We changed to a custom two-stage aerosol collector with an LLNLdesigned virtual impactor preconcentrator followed by the SASS 2000 wetted-wall cyclone collector. This arrangement was selected to maximize the collector concentration rate by increasing the collection flow rate and keeping the collection volume small (see Eq. 4 above). The fluidics module was changed to a sequential injection analysis ${ }^{15,16}$ platform and processes developed with Global FIA Inc. (Fox Island, WA) based on their FloPro-4P. Key components of that system are a 1 $\mathrm{mL}$ syringe pump (Cavro, Tecan Systems Inc., San Jose, CA) and multiport selection valves (Cheminert, Valco Instruments Company Inc., Houston, TX). That equipment was selected for its ability to perform completely automated and complex fluid manipulations in the volume ranges of interest (down to about $5 \mu \mathrm{L}$ ) and to do so with very high reliability over hundreds of thousands of movements. Additional fluidics hardware specific to the Luminex assay included a small stirred tank for keeping the beads suspended and a bead trap (or "microsphere sequestering cell”) invented by Global FIA and used for sample preparation binding and labeling. ${ }^{17}$ Figure 4 shows the resulting APDS100 instrument in 2001. At this time, LLNL, in collaboration with Tetracore Inc. (Gaithersburg, MD), had also succeeded in developing multiplexed Luminex immunoassays for biological threat agents. ${ }^{9}$ 


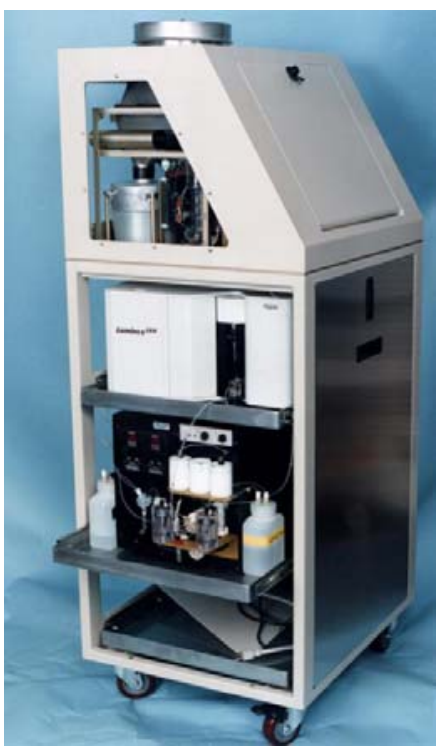

Figure 4. The APDS100 instrument in a stand-alone arrangement in 2001. The major subsystems of aerosol collector (top shelf), Luminex reader (second shelf), and fluidics module (third shelf) can be seen.

Later in 2001, there were shifts in the execution of the DOE program due to the September 11 plane attacks and the October anthrax attacks, with increased focus on transitioning to field use. The APDS team conducted a brief off-site operational exercise in late 2001; ran an integrated test with chamber releases of biological threat agent simulants in early 2002 at the University of Nevada, Las Vegas; and then demonstrated detection of aerosolized, viable threat agents in late 2002 at the Dugway Proving Ground. ${ }^{18}$ Figure 5 shows the APDS100 instrument in the foreground rearranged for the live-agent aerosol releases, where the collector was removed from the top of the chassis and placed in the Biological Safety Level 3 chamber. These chamber tests showed the end-to-end functionality of the system and its major subsystems: aerosol collector, fluidics module, Luminex reader, and multiplexed immunoassays. 


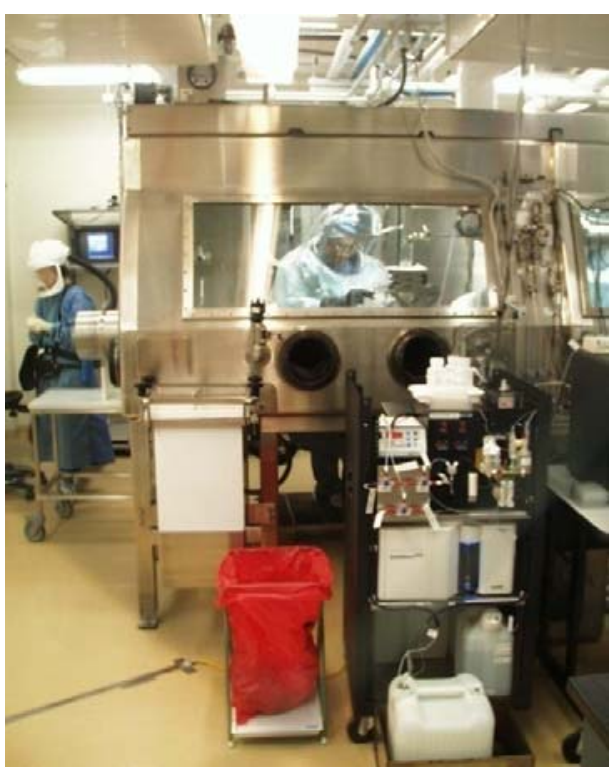

Figure 5. The APDS100 instrument running multiplexed immunoassays, attached to a Biological Safety Level (BSL) 3 aerosol chamber at Dugway Proving Ground for testing against live, aerosolized Bacillus anthracis and Yersinia pestis. The APDS instrument can be seen in the foreground in the BSL-2 laboratory; the aerosol collector was placed inside the BSL-3 chamber and pumped samples out for analysis. These chamber tests showed the end-to-end functionality of the system and its major subsystems: aerosol collector, fluidics module, Luminex reader, and multiplexed immunoassays. (Reprinted with permission from the journal Analytical Chemistry, Ref. 18, copyright 2003, American Chemical Society.)

In 2002, the Department of Homeland Security (DHS) was established, and the responsibility for civilian defense against biological terrorism was transferred from the DOE to the DHS. The DHS established the BioWatch Program in 2003 for environmental monitoring against terrorist attacks using biological threat agents. ${ }^{3}$ Subsequently, the APDS program transferred from the DOE to the DHS's Science and Technology Directorate, and later to the Systems Engineering Directorate and Office of Health Affairs for transition and operation. There were two major thrusts in the system development during this time: incorporating nucleic acid detection in addition to the multiplexed 
immunoassay, and establishing the required operational capabilities for field use. Nucleic-acid detection was integrated using a Flow-Through PCR module based on earlier PCR devices developed at LLNL with DoD and DOE funding. ${ }^{19,20}$ The flow-through format is ideally suited to the APDS's fluidics platform; the PCR module integrates essentially as a clamp-on reactor and detector on the existing valves and tubing. This next version of the instrument, dubbed the APDS150, used the multiplexed immunoassay as the primary test and real-time TaqMan PCR as a secondary, orthogonal test. This dual assay approach enabled a broad-spectrum but cost-effective test for initial detection of pathogens and proteins followed by a highly specific test for pathogen genetic sequences when necessary. The instrument was tested at Dugway Proving Ground in an aerosol chamber, where the instrument demonstrated autonomous detection of biological threat agents by multiplexed immunoassay with PCR confirmation. ${ }^{21}$ The APDS150 also began a series of extended field tests in this phase. ${ }^{22}$

The operational capabilities required for extended field use were developed and refined over a few years and several field tests and evaluations. One of the most important technical developments was establishing the APDS network and data viewer to enable remote, secure monitoring of biological and maintenance signals. Stable networking is necessary for fast and decisive response to biological alerts and is exercised constantly for tracking maintenance data by the minute. Other technical capabilities in the APDS150 period included: changing to a sealed, climate-controlled enclosure for dust, rain, and temperature tolerance; adding a heater to the aerosol collector for operation down to $-20^{\circ} \mathrm{C}$; adding a pre-separation stage to the aerosol collector inlet to cut down on dust; and incorporating battery backup to handle external power interruptions. The field operations were conducted in two airports, three subway stations, and other high-traffic and critical facilities. Over 20,000 field samples were autonomously collected, analyzed with multiplexed immunoassays, and reported. Figure 6 shows an APDS150 instrument during field testing. 


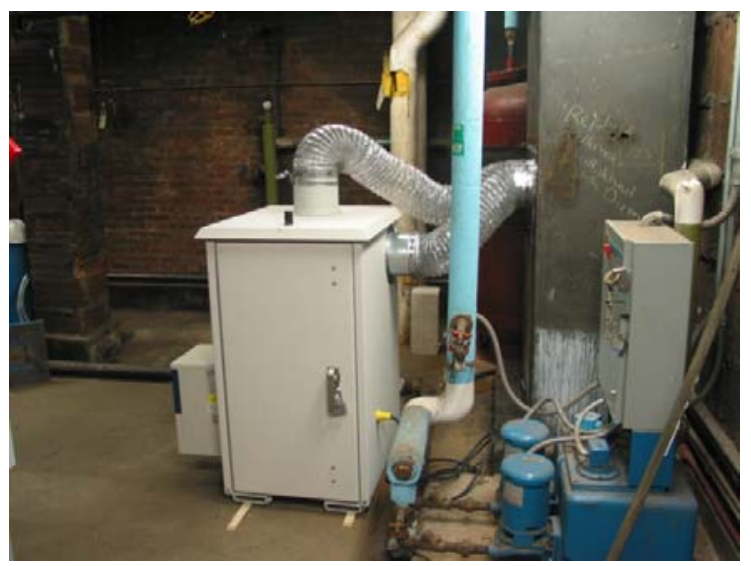

Figure 6. The APDS150 instrument is shown running multiplexed immunoassays with individual PCR confirmation, field testing in a ventilation system of a major transportation hub. This was part of a series of operational field tests and evaluations during which over 20,000 field samples were autonomously collected, analyzed with multiplexed immunoassays, secondarily analyzed with PCR when necessary, and reported over an encrypted network.

In addition to the technical advances in this period, there was a significant and sustained effort with the local stakeholders including public health, law enforcement, and facility representatives on operational aspects. This close collaboration was invaluable in figuring out how autonomous biological detection should fit in the daily operations and emergency responses of a city. The efforts included concepts of operations, response plans, usability assessments, feedback on features, and table-top exercises with key decision-makers.

\subsection{Recent development}

The final development steps to the current operational system were taken in 2006. The major change for this phase was integration of a multiplexed PCR assay for detection of nucleic acid using the Luminex platform. We extended earlier instrumentation work and assay development on multiplexed PCR and combined that with the field-ready APDS150 platform, thus developing the APDS300 instrument. The 
hardware for the assay (PCR module, Luminex reader, bead reservoir, and bead trap), and the reagent types (enzyme, primers, beads, and streptavidin-phycoerythrin) were already present in the instrument; what was required was a change in reagent composition and rigorous fluidics process development. The final result was an intricate process that required over 1,200 commands per report cycle, but that was robust enough to run unattended in the field. Figure 7 shows the schematic diagram for the subset of the fluidics that conducts multiplexed PCR with Luminex detection.

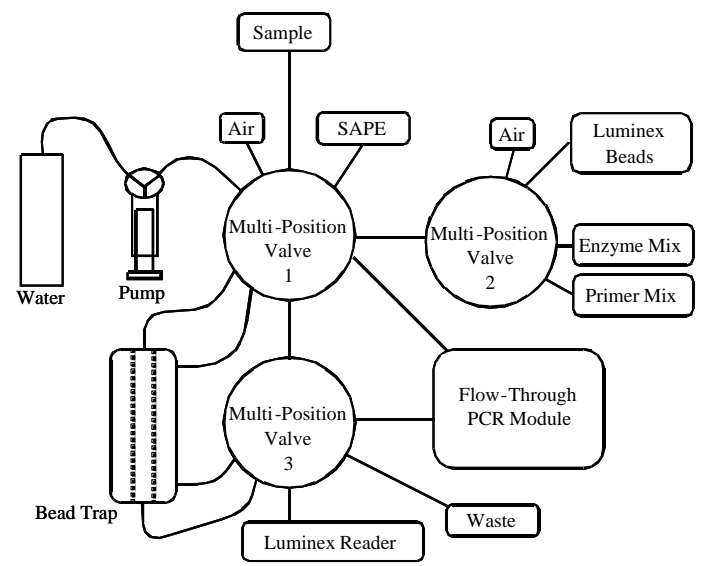

Figure 7. A schematic diagram of the APDS300 fluidics manifold that conducts multiplexed PCR, including sample preparation, by amplifying specific sequences using PCR, hybridizing amplicons to specific probes coupled to Luminex beads, labeling hybridized beads with streptavidin-phycoerythrin, and reading the resulting prepared sample in the Luminex microflow cytometer. The amplification and hybridization occur in the flow-through PCR module, and the labeling with washing occurs in the bead trap. (Adapted with permission from the journal Analytical Chemistry, Ref. 2, copyright 2008, American Chemical Society.)

The APDS300 was tested running a multiplexed PCR assay panel that had already been developed by LLNL for the DHS's BioWatch Program, tested at the CDC, and piloted in multiple Laboratory Response Network laboratories. One phase of the APDS testing was performed in a BSL-2 laboratory, where viable, unlysed, and cleaned B. anthracis spores and $Y$. 
pestis cells in liquid were used to compare the APDS multiplexed results to the manual process with TaqMan PCR. This was not an assay comparison but an integrated process comparison starting with the preparation step (Figure 1); the APDS process used the viable sample directly while the manual process included bead beating and ultrafiltration for sample preparation.

Figure 8 shows a comparison between the results for viable biological threat agent. The interpretation is complicated by the thresholds and detection algorithm described earlier, but generally speaking, samples with TaqMan Ct values less than 32 fully satisfied the detection algorithm, whereas samples with $\mathrm{Ct}$ values between 32 and 34 were fairly reliably detected by multi-reactive signatures, but not always. In contrast, samples with $\mathrm{Ct}$ values above 34 were not completely detected, although reactive signatures were present in all but the least concentrated sample. The cutoff for reliable TaqMan detection is a Ct of about 36, depending on the signature. There is room for improvement in the APDS process, and it was demonstrated that the addition of lysis to sample preparation would improve the sensitivity. 

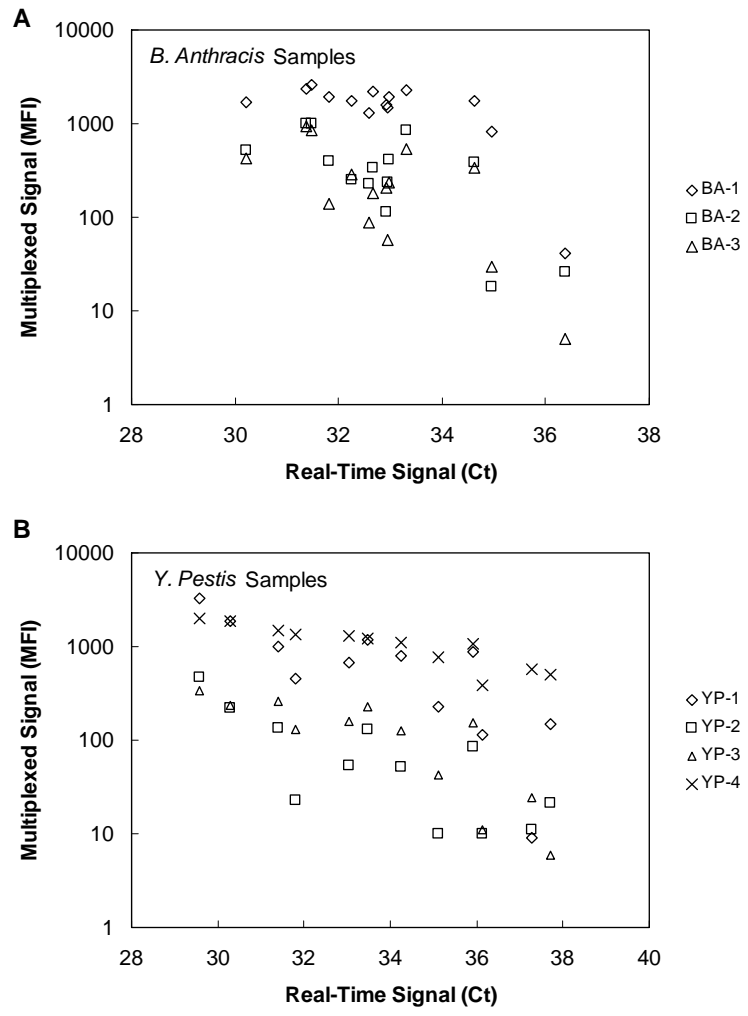

Figure 8. Comparison of the performance of APDS multiplexed analysis to a manual laboratory analysis for the detection of concentrations of B. anthracis (Panel A) and $Y$. pestis (Panel B). The APDS process introduced unlysed liquid sample directly into a multiplexed PCR assay, whereas the manual laboratory process introduces extracted and purified nucleic acids into single-plex TaqMan assays. The MFI values for each of the $B$. anthracis and $Y$. pestis signatures are shown for each sample concentration tested, represented as $\mathrm{Ct}$ values from real-time PCR analysis. (Reprinted with permission from the journal Analytical Chemistry, Ref. 2, copyright 2008, American Chemical Society.)

One of the multiple real-world testing environments for the APDS300 was a high-traffic subway station. The system remained in continuous operation, sampling $1700 \mathrm{~L}$ of air per minute for over two months, during which time 493 aerosol samples were analyzed. Figure 9 shows 
the results for all of the samples, with biological agent signals in Panels A, B, and C, and positive and negative control assays in Panel D. All of the assays yielded control MFI values within the specified ranges, with the exception of sample \#91 whose amplification positive control (PC) signal dropped to 135, which is below the acceptable lower limit of 200 for this control (Figure 9, Panel D). The assay on the next sample returned an acceptable PC MFI value without intervention. The signatures shown in the other panels (A, B, and C) have low baseline noise with the primary exceptions being BA-1 and YP-4 signatures, which are two of the more sensitive signatures in this multiplexed PCR assay. These results are expected and are consistent with the analysis thresholds and detection algorithms incorporating multiple signatures per agent. 


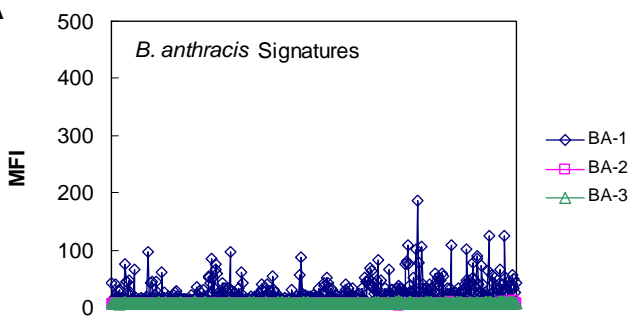

B

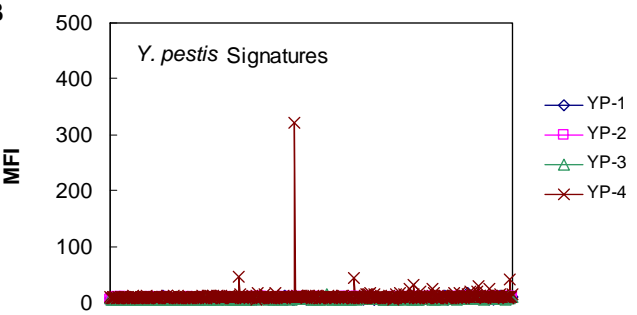

C
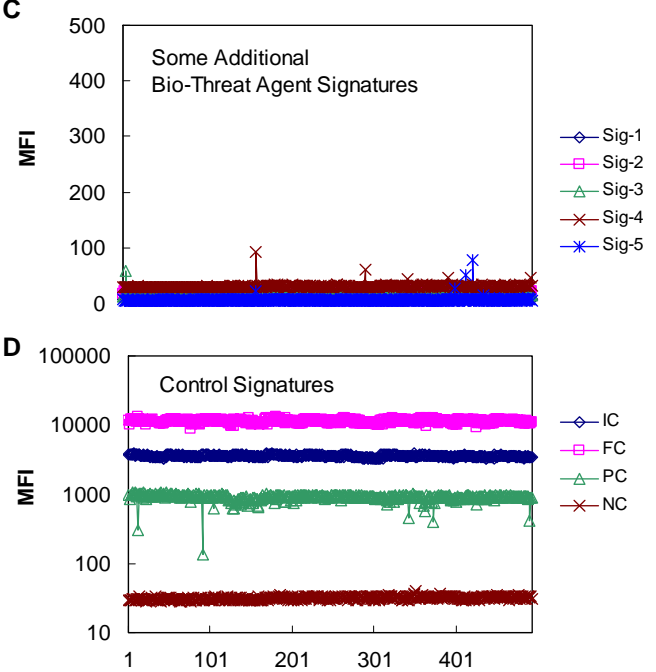

Figure 9. Performance of APDS in a high-traffic subway station. The APDS unit performed 493 multiplexed assays over a 2-month period. The MFI values for $B$. anthracis, $Y$. pestis, five selected additional bio-threat agent signatures, and the control signatures are shown in panels A, B, C, and D, respectively. (Reprinted with permission from the journal Analytical Chemistry, Ref. 2, copyright 2008, American Chemical Society.) 
The APDS300 instrument became the first actionable autonomous detector component of the U.S. Department of Homeland Security's BioWatch program. Figure 10 shows the APDS300 readied for operational use running multiplexed PCR assays. Compared to the early integration for proof of concept (Figure 4), the instrument is now less attractive but considerably hardened and more capable. The APDS300 as autonomous BioWatch "Generation 2.5" is operating at small scale. The DHS is entering a formal, full and open "Generation 3" competitive acquisition program for larger-scale autonomous BioWatch with an extensive test and evaluation process. ${ }^{24}$

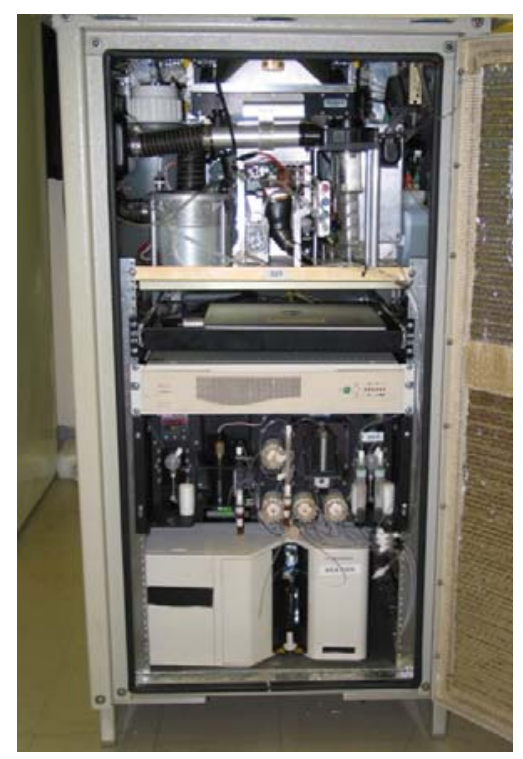

Figure 10. The APDS300 instrument running multiplexed PCR assays, readied for deployment for operational field use, with door open to show the internal equipment. The major subsystems of aerosol collector, uninterruptible power supply, fluidics module, and Luminex reader are visible from top to bottom, respectively. Compared to the early integration for proof of concept (Figure 4), the instrument is now less attractive but considerably hardened and more capable. 


\section{Critical Issues}

\subsection{Problems to be resolved}

As discussed above, the autonomous pathogen detection mission is uniquely demanding: continuous monitoring, multiple threat agents, high sensitivity, extremely low false-positive rates, and challenging environments. Years of field testing followed by a year of operational use have shown that APDS can perform the mission. The main areas for improvement are cost, speed, and sensitivity.

The current APDS acquisition cost is around $\$ 100,000$ per instrument, depending on the scale on which it is produced and which optional features are included. The true operation cost is less clear because the system is currently being transitioned to industrial operation, but it is probably over $\$ 100,000$ per instrument per year at small scale. This cost is acceptable for the highest-impact locations where many thousands of people can be protected, but limits the widespread use of the system.

The APDS multiplexed PCR report is issued about every two hours; the report cycle is about an order of magnitude faster than the standard manual process with daily filter collections. This speed is fast enough to help identify and effectively treat those who were exposed, and to prevent unexposed people from entering a contaminated area. However, if the speed could be increased by another order of magnitude so the delay was only a few minutes, this could protect people who are in the area while the release is occurring by getting them to evacuate before they inhaled an infectious dose.

Sensitivity is another area that can always use improvement. Especially for detection outdoors, where the air volumes are great and the area to monitor is large, better sensitivity gives the chance of detecting a release far from its source. However, there may be a limit to how far down the LOD should go: many of the biological threat agents are naturally present in the environment at low levels, and detection at that level should not lead to response actions. 


\subsection{Future outlook for progress}

LLNL and our industrial partner are engaged in several developmental efforts to improve the cost, speed, and sensitivity of the APDS process. For cost, we are streamlining the operation of the system and reducing the subsystem costs. This includes working with Luminex on a new reader platform tailored for field use in applications like the APDS. For speed and sensitivity, we are pursuing improvements in most of the processing steps covered earlier. Since the preparation time dominates, performing preparation in parallel for two samples can nearly halve the processing time.

Many other approaches to detecting biological threat agents have been described in the technical literature, but few of them make it to integrated field testing. Sometimes the approach is an improvement in one of the attributes of cost, speed, or sensitivity, but causes deterioration in one of the other of those attributes. Often, though, the main issue is that the technique cannot meet the mission's requirement for the rate of false positives to be only one every few years.

Beyond the current APDS mission, there are other related challenges ahead. One challenge is determination of threat agent viability, where the question is no longer whether the agent is present, but whether it is likely to cause infections. Another challenge is the detection of novel or emerging pathogens, which amounts to detecting an unknown and knowing that it is a threat. These lofty technical goals combined with the harsh reality of field operations will challenge workers in this area for some time to come. The importance of the mission makes it worthwhile.

\section{Acknowledgements}

The APDS program has been advancing for over a decade, and there have been many key contributors to the advances and successes. Most of them do not work on the project full time, but make critical contributions in their areas of expertise. The current contributors at LLNL include Tom Metz, Dean Hadley, Todd Corzett, Bruce Henderer, Vincent Riot, John Breneman, Elizabeth Wheeler, Chris Bailey, Todd Weisgraber, Shanavaz Nasarabadi, Christine Hara, Sally Hall, Staci Kane, Pejman 
Naraghi-Arani, and Jason Olivas, with additional support from Bill Benett, Dean Urone, Bob Paris, Kris Montgomery, and Julie Avila. Team members not mentioned above in the previous phase of the APDS150 and extensive field testing included Ben Hindson, Mary McBride, Julie Perkins, Dora Gutierrez, Dennis Imbro, Chris Spadaccini, Ujwal Sathyam, Candice Cook, Corey Chinn, Ramki Madabhushi, Sally Smith, Dave Cordes, Jack Regan, Stein Weissenberger, Wendy Wilson, Claudia Hertzog, and Erik Hofmann. Previous to that, other workers who took the project from proof-of-concept to APDS100 included Bill Colston, Rich Langlois, Steve Brown, Don Masquelier, Al Ramponi, Robert Johnson, Anne Marie Erler, Keith Burris, Pete Meyers, and Paul Sargis. The initial development was instigated by Don Prosnitz and conducted through its initial steps by Ray Mariella, Fred Milanovich, Robin Miles, Kodumudi Venkateswaran, and Les Jones.

There have also been many collaborators outside of LLNL who were critical to the development and successes: the CDC in the public sector and Northrop Grumman Corp. in the private sector are standouts. Other close industrial collaborations over the years have included Luminex Corp., Global FIA Inc., Research International Inc., Tetracore Inc., Radix BioSolutions Inc., and Biosearch Technologies Inc.

Critical phases of field testing were made possible by the New York City Police Department, Department of Public Health and Mental Hygiene, and Metropolitan Transit Authority; San Francisco Bay Area Rapid Transit and International Airport; Washington Metropolitan Area Transit Authority; Albuquerque International Sunport; and San Diego Air Pollution Control District.

Greatest thanks go to the local authorities including public health officials, facility and system operators, and law enforcement personnel who are involved in the current operational system. They cannot be named here, but their feedback enabled the transition from R\&D prototype to operational, national-security asset, and their expertise keeps the system running.

The APDS was initiated with LLNL internal seed funding, then largely funded by the U.S. Department of Energy and more recently by the U.S. Department of Homeland Security. We thank the DHS for their continued support and vision. Related work that contributed to the 
APDS development path was funded by the U.S. Department of Defense. This work was performed under the auspices of the U.S. Department of Energy by Lawrence Livermore National Laboratory in part under Contract W-7405-Eng-48 and in part under Contract DE-AC5207NA27344.

We apologize for any names that were omitted and contributions that were distorted.

This document was prepared as an account of work sponsored by an agency of the United States government. Neither the United States government nor Lawrence Livermore National Security, LLC, nor any of their employees makes any warranty, expressed or implied, or assumes any legal liability or responsibility for the accuracy, completeness, or usefulness of any information, apparatus, product, or process disclosed, or represents that its use would not infringe privately owned rights. Reference herein to any specific commercial product, process or service by trade name, trademark, manufacturer, or otherwise does not necessarily constitute or imply its endorsement, recommendation, or favoring by the United States government or Lawrence Livermore National Security, LLC. The views and opinions of authors expressed herein do not necessarily state or reflect those of the United States government or Lawrence Livermore National Security, LLC, and shall not be used for advertising or product endorsement purposes. The Department of Homeland Security's sponsorship of the production of this material does not constitute an endorsement of any products, services, policies, or activities of the authors.

\section{References}

1. J. P. Fitch, E. Raber, D. R. Imbro, Science 302, 1350 (2003).

2. J. F. Regan, A. J. Makarewicz, B. J. Hindson, T. R. Metz, D. M. Gutierrez, T. H. Corzett, D. R. Hadley, R. C. Mahnke, B. D. Henderer, J. W. Breneman IV, T. H. Weisgraber, and J. M. Dzenitis, Anal. Chem. 80, 7422 (2008).

3. D. A. Shea and S. A. Lister, The BioWatch Program: Detection of Bioterrorism (Congressional Research Service Report No. RL 32152, 2003). Accessed online at http://www.fas.org/spg/crs/terror/RL32152.html on March 31, 2008.

4. P. J. Meehan, N. E. Rosenstein, M. Gillen, R. F. Meyer, M. J. Kiefer, S. Deitchman, R. E. Besser, R. L. Ehrenberg, K. M. Edwards, K. R. Martinez, CDC MMWR Recommendations Rep. 53 (RR07), 1 (2004). 
5. T. P. Christie, Director, U. S. Department of Defense Operational Test \& Evaluation, Annual Report FY 2003. Accessed online at http://www.globalsecurity.org/military/library/budget/fy2003/fy03_DOTE_An nual_Report.pdf on April 12, 2008.

6. J. Carrano, T. Jeys, D. Cousins, J. Eversole, J. Gillespie, D. Healy, N. Licata, B. Loerop, M. O’Keefe, A. Samuels, J. Schultz, M. Walter, N. Wong, B. Billotte, M. Munley, E. Reich, and J. Roos, Chemical and Biological Sensor Standards Study (Defense Advanced Research Projects Agency publication, 2004).

7. J. A. Rice, Mathematical Statistics and Data Analysis (Wadsworth and Brooks/Cole, Belmont, 1987), p. 39.

8. N. R. Beer, B. J. Hindson, E. K. Wheeler, S. B. Hall, K. A. Rose, I. M. Kennedy, B. W. Colston, Anal. Chem. 79, 8471 (2007).

9. M. T. McBride, S. Gammon, M. Pitesky, T. W. O’Brien, T. Smith, J. Aldrich, R. G. Langlois, B. Colston, K. S. Venkateswaran, Anal. Chem. 75, 1924 (2003).

10. W. J. Wilson, A. M. Erler, S. L. Nasarabadi, E. W. Skowronski, P. M Imbro, Mol. Cell. Probes 19, 137 (2005).

11. J. W. Jacobson, K. G. Oliver, C. Weiss, and J. Kettman, Cytometry A 69, 384 (2006).

12. K. B. Olson, Emerg. Infect. Dis. 5 (4), 513 (1999).

13. R. P. Mariella Jr., G. van den Engh, D. Masquelier, and G. Eveleth, Cytometry 24, 27 (1996).

14. R. P. Mariella Jr., Z. Huang, and R. G. Langlois, Cytometry 37, 160 (1999).

15. J. Ruzicka and G. D. Marshall, Anal. Chim. Acta 237, 329 (1990).

16. C. E. Lenehan, N. W. Barnett, and S. W. Lewis, Analyst 127, 997 (2002).

17. B. J. Hindson, S. B. Brown, G. D. Marshall, M. T. McBride, A. J. Makarewicz, D. M. Gutierrez, D. K. Wolcott, T. R. Metz, R. S. Madabhushi, J. M. Dzenitis, B. W. Colston Jr., Anal. Chem. 76, 3492 (2004).

18. M. T. McBride, D. Masquelier, B. J. Hindson, A. J. Makarewicz, S. B. Brown, K. Burris, T. R. Metz, R. G. Langlois, K. W. Tsang, R. Bryan, D. A. Anderson, K. S. Venkateswaran, F. P. Milanovich, B. W. Colston Jr., Anal. Chem. 75 5293, (2003).

19. P. Belgrader, W. Benett, D. Hadley, J. Richards, P. Stratton, R. Mariella Jr., and F. Milanovich, Science 16, 5413 (1999).

20. P. Belgrader, C. J. Elkin, S. B. Brown, S. N. Nasarabadi, R. G. Langlois, F. P. Milanovich, B. W. Colston Jr., Anal. Chem. 75, 3446 (2003).

21. B. J. Hindson, M. T. McBride, A. J. Makarewicz, B. D. Henderer, U. S. Setlur, S. M. Smith, D. M. Gutierrez, T. R. Metz, S. L. Nasarabadi, K. S. Venkateswaran, S. W. Farrow, B. W. Colston Jr., J. M. Dzenitis, Anal. Chem. 77, 284 (2005).

22. B. J. Hindson, A. J. Makarewicz, U. S. Setlur, B. D. Henderer, M. T. McBride, J. M. Dzenitis, Biosens. Bioelectron. 20, 1925 (2005).

23. B. J. Hindson, D. M. Gutierrez, K. D. Ness, A. J. Makarewicz, T. R. Metz, U. S. Setlur, W. J. Benett, J. M. Loge, B. W. Colston Jr., P. S. Francis, N. W. Barnett, and J. M. Dzenitis, Analyst 133, 248 (2007). 
24. U.S. Department of Homeland Security, Procure and Deploy an Autonomous Biodetection System Called Gen 3 BioWatch, draft solicitation number HSHQDC-09-R-00045D. Accessed online at https://www.fbo.gov/ on $2 / 20 / 2009$. 